\title{
Prácticas escolarizadas de escritura en estudiantes de Letras hispánicas. Una descripción de la formación de sujetos letrados
}

\section{In-school writing practices with students of Spanish Literature: a description of the training of literate subjects}

DOI: https://doi.org/10.32870/dse.v0i23.963

\section{Luz Eugenia Aguilar González* Gilberto Fregoso Peralta**}

\begin{abstract}
Resumen
Este artículo describe algunas prácticas escolarizadas de escritura realizadas en la educación básica y media que efectuaron estudiantes de la Licenciatura en Letras Hispánicas. El objetivo es describir las prácticas de escritura más frecuentes durante dicha etapa escolar, así como el significado que los estudiantes les otorgan al incorporarlas en su proceso de aprendizaje y que los han formado como sujetos letrados. El trabajo toma sus bases teóricas de los Nuevos Estudios de Literacidad (NEL) y de los estudios de Escritura a través del Curriculum (WAC).

Desde estas perspectivas se construyeron tres categorías analíticas: actividades escolares, revisión de la escritura y oralización de la escritura. Con ellas se diseñó un cuestionario de 15 preguntas de dos tipos: cerradas dicotómicas y abiertas. Las preguntas cerradas permiten conocer la frecuencia de tipos de escritos y de prácticas escolares específicas, y las abiertas ayudan a comprender la significación de los estudiantes sobre dichas prácticas. Se aplicó a un total de 67 estudiantes de primero, octavo y noveno semestres. Los resultados muestran que la escuela realiza prácticas letradas diversas y que están transitando de la alfabetización a la literacidad. Los estudiantes significan la escritura como una práctica discursiva, lo que los caracteriza como sujetos letrados.
\end{abstract}

Palabras clave: escritura - literacidades - prácticas letradas - revisión - oralización.

\section{Abstract}

This article describes some in-school writing practice activities carried out by undergraduate students of Spanish Literature in basic and middle school. The aim is to describe the most frequent writing practices

\footnotetext{
* Doctora en educación. Miembro del SNI y del COMIE. Cuerpo Académico Consolidado "Lenguaje, literatura y literacidad". Líneas de investigación: Literacidad académica y comunicación educativa. Profesora-investigadora en el Departamento de Letras, Universidad de Guadalajara. México. aguilar.luzeugenia@gmail.com

** Doctor en Ciencias. Líneas de investigación: Lenguaje, literatura y literacidad. Profesor-investigador adscrito al Centro Universitario de Los Altos. Universidad de Guadalajara, México. fepg@hotmail.com
} 
during basic and middle education as well as the meaning given to them by students when they incorporate them into their learning process and become literate persons. The theoretical foundations of our work are those of the New Literacy Studies (NLE), as well as experiences from the Writing across the Curriculum (WAC) movement.

Based on these approaches, we designed three analytic categories: school activities, feedback in writing, and the oralization of writing. These categories were used to design a questionnaire of 15 questions, including both dichotomous questions and open questions. Closed-ended questions allowed us to know the frequency of types of writing and specific scholar practices. Open-ended questions helped us to understand the significance given by the students to these practices. The questionnaire was applied to $67 \mathrm{stu}$ dents from first, eighth and ninth semester. The results show that the school carries out a variety of literacy practices and that it is moving from basic to full literacy. For the students, writing is a discursive practice which characterizes them as literate persons.

Key words: writing - literacies - literacy practices - feedback - oralization.

\section{Acercamiento previo}

Este trabajo describe algunas prácticas de escritura de estudiantes de la Licenciatura en Letras Hispánicas, anteriores a su ingreso al pregrado y el significado que ellos les asignan. Los estudios sobre escritura analizan los procesos de adquisición y desarrollo de la lectura y la escritura en las primeras etapas de la alfabetización, o bien los problemas y retos de la escritura académica en el ámbito escolar. Actualmente se investiga sobre los problemas de escritura de los estudiantes en los distintos niveles educativos. Sin embargo, pocos son los estudios sobre estudiantes con alta competencia escritural (Flórez Romero et al., 2005; Martínez, 1997). Esta investigación viene a cubrir parte de este espacio vacío dentro de los estudios de escritura, además, ayudará a comprender mejor las actividades escolares que podrían fortalecer la competencia escrita desde la experiencia de los propios sujetos. De esta manera, los resultados de este trabajo se pueden relacionar con distintas propuestas de enseñanza de la lengua y estudios descriptivos sobre procesos de aprendizaje de la escritura, a la par de comprender las razones por las que pueden funcionar para cierto tipo de estudiantes.

Los estudiantes de la Licenciatura en Letras Hispánicas de la Universidad de Guadalajara, en su mayoría, tienen alta competencia escritural. Es importante subrayar que por su interés en el lenguaje, los estudiantes de letras tienen facilidad para acercarse a los procesos de lectura y escritura; pero, si hacemos un lado esta variable, lo que nos interesa es identificar las prácticas escolares de escritura que recuerdan haber realizado en niveles educativos previos a la licenciatura y que pudieron haber contribuido a su formación como sujetos letrados. Para completar la explicación sobre sus prácticas escolares y la relación con su competencia escritural, indagamos en la manera en que ellos significan algunas de estas prácticas escolares como parte de su proceso de aprendizaje. 
Las características particulares de estudiantes que estuvieron inmersos en procesos escolares similares pueden tener distintos niveles de desarrollo en sus competencias y habilidades de escritura. Por otros estudios sabemos de las dificultades de los estudiantes de pregrado cuando se enfrentan a la escritura académica (Carlino, 2004). De ahí la inquietud de conocer ¿cuáles fueron las prácticas de escritura en educación básica y media superior de los estudiantes de Letras hispánicas que contribuyeron a formarlos como escritores competentes y los significados que les otorgan dentro de su proceso formativo? La descripción de estas prácticas, a su vez, permite definir a los estudiantes como sujetos letrados, lo que significa que hablan y piensan por sí mismos el mundo (Hernández-Zamora, 2019). Pretendemos no quedarnos solo en la descripción de las prácticas, sino comprender procesos más complejos que los mismos estudiantes manifiestan sobre su proceso educativo.

De los pocos estudios encontrados sobre escritura exitosa está el de Freedman (1987), quien realiza su investigación en dos grupos de noveno semestre en la clase de escritura. Concluye que los grupos de estudiantes se ayudan a responder a su propio trabajo de escritura por medio del trabajo grupal puesto que es una manera de tener presente a la audiencia. Por otra parte, los estudiantes usan los grupos para discutir el contenido de su escritura, aunque no tengan un propósito explícito para hacerlo. Ello les permite pensar colaborativamente (Freedman en Vargas Franco, 2015: 46).

Por su parte, los Nuevos Estudios sobre Literacidad (NEL) presentaron conceptualizaciones teóricas y acercamientos empíricos distintos a los estudios de la lectoescritura o de la alfabetización. El aporte fundamental de los NEL es considerar la lectura y la escritura como prácticas sociales. Desde este planteamiento, los aportes básicos de los NEL se resumen por sus tres hipótesis fundamentales: 1) Shirley Brice Heath encontró en sus estudios que las personas participan constantemente en eventos de literacidad que pueden ser de índole oral o escrita, o bien pasar de la oralidad a la escritura. De esta forma, no tiene sentido separar los eventos orales de los escritos y vincular conceptualmente a la escritura solamente con la alfabetización, sino que el punto medular es identificar cómo se usa la escritura, con quién y en qué contexto (Hernández-Zamora, 2019). 2) Para B. Street, aprender a leer y a escribir no es solo la adquisición de una habilidad, sino de diversas literacidades, lo que es posible al participar en distintas prácticas sociales (Hernández-Zamora, 2019). Y 3) Que las prácticas letradas no pueden separarse de los discursos, entendidos estos como "sistemas sociales de significado que median la constitución de posiciones del sujeto" (Hernández-Zamora, 2019: 367).

De esta manera, la enseñanza de la escritura se inscribe en distintas prácticas situadas, mismas que fortalecen el compromiso de las personas, construyen identidades y promueven las relaciones sociales (Zavala, 2011). Desde esta perspectiva, la escritura vincula el entorno con las preocupaciones de los sujetos y con el acto de escribir dentro de espacios escolares (Aguilar, Fregoso, 2013). En el ámbito escolar, la escritura se practica intencionalmente como: a) una forma de adquirir 
conocimiento (con la elaboración de resúmenes, síntesis o monografías); b) como una forma de organizar y demostrar conocimientos (ensayos, reflexiones, relatos) y c) como una manera de acercarse a la realidad, de problematizarla y de actuar en un entorno determinado para alcanzar un fin desde una perspectiva disciplinar (proyectos de trabajo, reportes de investigación).

Desde esta perspectiva, si se considera a la escritura como una habilidad situada en un contexto particular, con un sentido y con una finalidad dada, aplicada a situaciones concretas, la clasificación de la escritura como una competencia genérica omite un acercamiento desde distintas literacidades que incluyan las prácticas sociales y la mediación discursiva de los ámbitos letrados.

En este sentido, la escritura dentro de la escuela y organizada en los contenidos curriculares, podría no estar suficientemente contextualizada como para desarrollarse más sofisticadamente. La práctica de la escritura dentro de las instituciones escolares es definida por algunos autores como escritura académica, pero esta definición debería trascender estos límites pues la escritura académica no se basa solo en las tareas que realiza un estudiante, sino que también contiene ideas que se "fundamentan en saberes expertos a partir de un conjunto de argumentos previamente establecidos por cada comunidad académica en particular" (Cervantes Velázquez, 2015: 47). Sin embargo, las prácticas de escritura que se realizan en los niveles previos a la universidad son pocas y se usan principalmente como estrategias de aprendizaje. Ya en la universidad, en el mejor de los casos, se practica la lectura y la escritura para la recuperación de ideas, para demostrar conocimientos y para la investigación, prácticas que constituirán la formación disciplinar del estudiante; sobre este último aspecto, hay escasa reflexión sustentada en investigaciones sobre los procesos de construcción de conocimiento y de su uso en situaciones prácticas particulares en la práctica profesional.

Durante el periodo escolarizado se ejercitan distintas habilidades básicas para la escritura, pero para dominar la competencia es necesaria la escritura en contexto, lo que se ha definido como práctica situada. Desde un enfoque culturalista y como herramienta epistémica, la escritura fomenta las actividades analíticas, la delimitación clara de un tema de estudio; permite realizar juicios críticos, argumentados, además de practicar el pensamiento basado en la razón (Cervantes Velázquez, 2015). A pesar de ser esta una propuesta actual, desconocemos si la escritura en la escuela se acerca a estos planteamientos.

Por otra parte, la producción escrita y la comprensión lectora son procesos psicosociolingüísticos en donde se aplican las competencias lingüísticas, cognoscitivas y comunicativas, además de un "dominio sobre el contenido del mensaje y sobre el contexto social de comunicación" (Fraca de Barrera, 1997: 109). Si atendemos a estos supuestos, cuando se analiza el problema de la escritura en la escuela debemos considerar tanto aspectos lingüísticos como de otros órdenes, pues está claro que los acercamientos analíticos centrados solo en la pedagogía son insuficientes.

Desde este plateamiento, abordar la enseñanza de la escritura en todos estos frentes es 
complejo. Cuando los estudiantes llegan a la universidad, en su mayoría, no dominan la escritura académica. Quienes lo logran, han realizado tanto prácticas personales como escolares que les permiten ser exitosos en esta esfera, además de sus entornos culturales. Este acercamiento al objeto de estudio nos ubica dentro de una de las preocupaciones teóricas a resolver de los NEL. El acercamiento empírico para describir las prácticas escolares de escritura nos ayudará a caracterizar a los estudiantes de Letras hispánicas como sujetos letrados que hablan y piensan por sí mismos en su estar en el mundo.

\section{Los estudiantes de Letras hispánicas}

Los jóvenes que ingresan a la licenciatura en Letras hispánicas tienen preferencia por los fenómenos de la lengua y la literatura, además de una inclinación especial hacia las manifestaciones artísticas. Sobra decir que tienen amplias lecturas y, en algunos casos, interés especial por la escritura creativa. Si se revisan los puntajes que obtuvieron los estudiantes en la Prueba de Aptitud Académica (PAA) ${ }^{1}$ para su ingreso a la Universidad de Guadalajara (UdeG) en los calendarios de $2013 \mathrm{~A}^{2}$ a $2018 \mathrm{~A}$, los admitidos a la carrera de Letras hispánicas se encuentran en el tercer lugar de puntajes máximos (193.30 puntos) entre las carreras que ofrece el Centro Universitario de Ciencias Sociales y Humanidades (CUCSH), debajo de Derecho y Relaciones Internacionales. Sin embargo, por promedio de puntajes se encuentra en primer lugar (174.54 puntos) de un total general de 159.92. De todas las carreras que ofrece la UdeG, se encuentra en el sexto lugar del puntaje más bajo obtenido (156.98). ${ }^{3}$ El puntaje más bajo en otras carreras es: Medicina (173.83), Ingeniería biomédica (162.4), Arquitectura (159.21), Odontología (159.14) y Administración financiera (157.80) (véase tabla 1). ${ }^{4}$

1 La PAA evalúa las habilidades y los conocimientos necesarios para el trabajo académico universitario. Su objetivo es "predecir, junto con otros criterios, el éxito en el primer año de estudios superiores". En su página aparece que universidades públicas y privadas "confían en este instrumento por su rigor psicométrico, pertinencia cultural y valor predictivo" https://latam.collegeboard.org/paa/que-es-la-paa 2 En la Universidad de Guadalajara tenemos dos semestres divididos en: semestre de invierno (calendario A) y el de verano (calendario B).

3 Datos con base en los resultados de la aplicación de la PAA del 2013A (calendario de invierno) al 2018B (calendario de verano). Fuente: La Gaceta Universitaria. Órgano de comunicación de la Universidad de Guadalajara.

4 Datos obtenidos de las publicaciones de los dictámenes en La Gaceta Universitaria. Órgano de comunicación de la Universidad de Guadalajara.

Diólo@os sobre Educación 
Tabla 1. Puntajes de la PAA para el ingreso a la Universidad de Guadalajara de $2013 \mathrm{~A}$ a $2018 \mathrm{~A}$

\begin{tabular}{|l|l|l|l|}
\hline \multicolumn{2}{|c|}{ Por mínimo de calificación } \\
\hline \multicolumn{2}{|c|}{} \\
\hline Centro Universitario
\end{tabular}

\begin{tabular}{|l|l|lr|}
\hline \multicolumn{3}{|c|}{ Por Calificación promedio } \\
\hline \multicolumn{2}{|l|}{} \\
\hline Centro Universitario \\
\hline CUAAD
\end{tabular}

\begin{tabular}{|c|c|c|c|}
\hline \multicolumn{4}{|c|}{ Por calificación máxima } \\
\hline Centro Universitario $\equiv \nabla_{x}$ & $\bar{E} \equiv$ & Etiquetas de fila & Máx. de Calificación \\
\hline CUAAD & Temático & Abogado & 194.8389 \\
\hline CUAltos & Regional & & \\
\hline CUCBA & & & \\
\hline & & & \\
\hline CUCEA & 決陑 & Antropología & 188.8333 \\
\hline CUCEI & 2013B & LIDIFLE & 187.7222 \\
\hline $\mathrm{CUCi}$ & 2014A & Abogado semi & 186.2778 \\
\hline CUCosta & 2014B & LIDILE & 185.6656 \\
\hline CUCS & $2015 A$ & $\frac{\text { Filosofia }}{\text { Total general }}$ & $\begin{array}{r}185.1978 \\
194.8389\end{array}$ \\
\hline CUCSH & $2015 B$ & & \\
\hline & $2016 A$ & & \\
\hline
\end{tabular}

Fuente: Elaboración de los autores.

En los inicios del movimiento “Escribir a través del Currículum” (WAC por sus siglas en inglés, Bazerman et al., 2016) se plantea la pertinencia de que en los primeros semestres de las carreras haya materias curriculares para desarrollar habilidades de escritura específicas del área. Ello se siguió como premisa en los diseños curriculares de distintas latitutes. Por ejemplo, en el CUCSH 
varias carreras compartieron hasta 2019 la materia de Expresión oral y escrita, cuya finalidad es mejorar las habilidades de expresión de los estudiantes, en particular en su área disciplinar. En 2019, la Licenciatura en Letras Hispánicas realizó una reforma al plan de estudios y separó los contenidos de expresión oral y expresión escrita en dos asignaturas, adscribiéndose a un enfoque más disciplinar -para lograr competencias profesionales específicas en los egresados-que a un enfoque de desarrollo de habilidades generales. Por su naturaleza, a lo largo de la licenciatura en Letras hispánicas, a los alumnos se les solicitan trabajos escritos de diversa índole. A medida que avanzan en la ruta curricular, los trabajos escritos preferidos por los profesores son los ensayos, artículos y reportes de investigación ${ }^{5}$ (Casillas Padilla, 2018).

En un estudio que evalúa textos escritos de los estudiantes de Letras hispánicas (Margarito, Quezada, Aguilar, 2013) la escritura de 50\% de los estudiantes se caracteriza por tener cohesión y coherencia, además de respetar la estructura discursiva que cumple con los cánones de los trabajos académicos. Los problemas de escritura detectados son: de estilo, de subordinación y de uso riguroso de alguno de los sistemas de citación; además aparecen rasgos de oralidad y problemas de puntuación. Tanto en este estudio como en una entrevista con empleadores ${ }^{6}$ de egresados en Letras hispánicas, los resultados coinciden en que la escritura está marcada por rasgos literarios, lo que para algunos espacios profesionales es un problema, pues se pierde la objetividad o las características textuales solicitadas para productos particulares o contenidos producidos, pero para otros espacios laborales puede ser una ventaja, pues el profesionista en Letras hispánicas es innovador y creativo. Esta ventaja ha permitido que los egresados se coloquen en empresas de medios y en la mercadotecnia como generadores de contenidos. ${ }^{7}$

\section{Metodología}

La presente investigación toma los principios de los NEL en cuanto a que consideramos la escritura como una práctica social situada, que incluye otras literacidades y que está mediada por divesos discursos. Desde el enfoque educativo, se incluye dentro de los estudios de la escritura a través del currículum. Estos trabajos establecen un vínculo entre la lectura y la escritura académica. La WAC reconoce la escritura como una actividad que propicia el aprendizaje centrado en el estudiante. El principio que une a los NEL y los de la WAC es el análisis de la forma en que la escritura establece conexiones con el material de estudio y el pensamiento disciplinar (Bazerman et al., 2016), así como que la escritura se considera una herramienta epistémica, continua y transversal que permite el aprendizaje durante el proceso educativo.

5 Además de la tesis citada, recuperamos estos mismos datos del encuentro con maestros y estudiantes realizado para la actualización curricular en diciembre de 2016.

6 Este encuentro se realizó para recabar datos para la actualización curricular, en diciembre de 2016.

7 Datos proporcionados por los empleadores (nota anterior). También se puede revisar en el buscador de empleos: https://www.indeed.com. $\underline{\mathrm{mx}}$ en el cual los empleadores prefieren a los licenciados en letras para puestos como gestores y generadores de contenidos.

Diálo oos sobre Educación 
En esta revisión se seleccionaron algunos conceptos teóricos y resultados de otras investigaciones sobre las prácticas de escritura de estudiantes. Con ellos construimos categorías analíticas que permitirán describir las prácticas de escritura en educación básica y media superior de los estudiantes de letras hispánicas y que contribuyeron a formarlos como escritores competentes. En los siguientes puntos se explica cada una de ellas.

\section{Actividades escolares}

En esta categoría se indagó sobre el texto más solicitado por los profesores durante la vida académica de los estudiantes, desde primaria hasta preparatoria, sobre el trabajo en taller con los textos y lo que fue más significativo para ellos. Conocer el tipo de texto más utilizado permite comprender la perspectiva educativa predominante dentro del proceso educativo; por otra parte, ello ayuda a caracterizar las prácticas letradas que definen las concepciones de la enseñanza de la escritura. Una clasificación útil para la interpretación de los resultados es la que propone Ramírez Silva (2012), quien diferencia los textos académicos de los escolares. Los textos escolares son los que se usan para aprender en las asignaturas, son de uso personal aunque, en ocasiones, los docentes los solicitan para verificar que se está logrando el aprendizaje o como una forma de control para la evaluación. Los textos académicos implican un conjunto de estrategias para la redacción, como la investigación previa, el empleo del lenguaje de la disciplina y la formulación y argumentación de una tesis de escritura. También pueden incluirse los análisis, la opinión personal, la crítica y otras narraciones. El trabajo de los textos en taller como una práctica escolar, permite analizar el trabajo colaborativo. Además de que es una actividad que se ha incorporado dentro de los planes y programas de la educación básica (Secretaría de Educación Pública, 2011).

\section{Revisión de la escritura}

Se parte del supuesto que para alcanzar la competencia de escritura, los profesores tienen que vigilar el proceso de producción. Uno de los momentos del proceso es la revisión de los trabajos. Se prefiró analizar la revisión del escrito sobre la planeación, no porque aquella tenga mayor importancia, sino porque consideramos que el proceso de revisión implica una reflexión profunda sobre distintos aspectos, tales como los lingüísticos y discursivos, además de ser una actividad compartida (entre el profesor y los estudiantes) y colaborativa (entre el grupo de pares). Asimismo, la revisión se relaciona con la motivación, pues el estudiante puede evaluar sus aciertos y errores y sentirse seguro para avanzar en su proceso. Por otro lado, el alumno se siente atendido por el profesor y la retroalimentación sobre su rendimiento permite que la actividad que está realizando sea interesante para así alcanzar la meta que se le propone (Soriano, 2001). Por su parte, la revisión entre pares propicia la formación de comunidades discursivas que alientan el aprendizaje colaborativo (Molina-Natera, 2012). En suma, se analiza cómo la formación 
en la lectoescritura depende de una comunidad de hablantes que hacen que el texto signifique algo para los alumnos. Es uno los principios de los NEL (Heath cit. por Hernández-Zamora, 2019).

\section{Oralización de la escritura}

La separación entre la oralidad y la escritura fue una hipótesis inicial de la alfabetización académica. Sustentaba que la oralidad y la escritura son habilidades separadas. Fue llamada la gran división oralidad-escritura, defendida por Jack Goody e lan Watt (1968) y por Walter Ong (1982) (en Hernández-Zamora, 2019). Por trabajos empíricos se comprobó que era un acercamiento erróneo el afirmar que de "la escritura en sí misma promoviera el pensamiento abstracto y lógico, el razonamiento analítico, etc." (Cole, 1981 en Hernández-Zamora, 2019: 366). Investigaciones posteriores demostraron que la interacción entre la oralidad y la escritura influye en el aprendizaje pues los eventos de literacidad son inseparables de las prácticas sociales. En el ámbito escolar, este ir y venir entre la oralidad y la escritura permite compartir y discutir conocimientos y procesos de aprendizaje. Es una estrategia que fija la práctica letrada. La escritura ayuda a retener, clarificar y organizar conocimientos, y la oralización de la escritura puede servir para aclarar y construir nuevos conocimientos (Bazerman et al., 2016). En el uso escolar hay una hibridación entre lo oral y lo escrito, que no se refiere a la lectura en voz alta o a las exposiciones orales, sino a aquella complementariedad que permite reflexionar y desarrollar procesos de aprendizaje complejos como los necesarios en la escritura académica. Ello impulsa la salida de la autoreferencialidad discursiva y mejora los procesos de reflexión, argumentación y comunicación. Se establece un continuum entre la oralidad y la escritura como una forma discursiva integrada y no como dos procesos distintos. Asimismo, responde a los principios de literacidad al incorporar diversas prácticas de interacción social dentro del espacio escolar y marcar la posición del escritor frente a su producto escrito.

Con estas categorías se diseñó un cuestionario con 15 preguntas de dos tipos: abiertas y cerradas dicotómicas (véase anexo). Las preguntas cerradas nos ayudaron a cuantificar las prácticas educativas recurrentes de niveles anteriores. Las preguntas abiertas permitieron matizar los resultados cuantificados y lograr una explicación más completa de la significación de las prácticas. Las preguntas se distribuyeron como se indica en el cuadro siguiente:

Cuadro 1. Distribución de la cantidad de preguntas en cada categoría

\begin{tabular}{|l|l|l|}
\hline $\begin{array}{c}\text { Actividades escolares } \\
\text { de la escritura }\end{array}$ & \multicolumn{1}{|c|}{$\begin{array}{c}\text { Revisión de la } \\
\text { escritura }\end{array}$} & \multicolumn{1}{c|}{$\begin{array}{c}\text { Oralización de la } \\
\text { escritura }\end{array}$} \\
\hline $\begin{array}{l}\text { 4 preguntas abiertas } \\
2 \text { cerradas dicotómicas }\end{array}$ & $\begin{array}{l}\text { 2 preguntas abiertas } \\
\text { 1 cerrada dicotómicas }\end{array}$ & $\begin{array}{l}\text { 2 preguntas abiertas } \\
\text { 4 cerradas dicotómicas }\end{array}$ \\
\hline
\end{tabular}

Fuente: elaboración de los autores. 
El cuestionario se aplicó a 67 estudiantes: 30 de primero y 37 de octavo y noveno semestres. Los de primero se seleccionaron por tener más fresca la experiencia en los niveles anteriores. Estos estudiantes no tienen todavía la formación en el área de lengua, por lo cual sus respuestas no están mediadas por la metareflexión, que implica el conocimiento de base sobre aspectos lingüísticos, pragmáticos o semánticos, y que son parte de los saberes de los egresados en Letras hispánicas. En este nivel se aplicó a todos los estudiantes del grupo con la ayuda de uno de sus profesores. La aplicación a los estudiantes de semestres más avanzados se realizó para tener un espectro más amplio de las respuestas. Los estudiantes participaron voluntariamente y el cuestionario se aplicó de forma individual cuando el estudiante aceptaba participar en el estudio. En este grupo, en las respuestas abiertas encontraremos reflexiones mediadas por sus conocimientos sobre lengua. En promedio, respondieron el instrumento en 10 minutos.

Una vez que tuvimos las respuestas, se pasaron todas a hojas de cálculo. Las respuestas a las preguntas cerradas se analizaron a partir de las frecuencias. Las respuestas de las preguntas abiertas se procesaron a partir de categorizaciones conceptuales. De ahí, se formularon hiperónimos que surgieron de la recurrencia de conceptos y significados encontrados en las respuestas. Para su análisis, se emplearon algunos principios de la fenomenología, como el punto de vista del participante, los planteamientos conceptuales de las respuestas y su posible significado. Así pudimos plantear estructuras universales para explicar y aprehender las experiencias de los estudiantes con la escritura (Hernádez Sampieri, Fernández-Collado, Baptista Lucio, 2006). En seguimiento a la sistematización de datos, para codificar la información de las respuestas abiertas se asignó un número para cada alumno. Las citas textuales de las respuestas de los estudiantes se presentarán con la abreviatura $E$ (estudiante) y el número asignado para la codificación en la hoja de cálculo, quedando como: E1, E2, E3, etcétera.

\section{Resultados}

\section{Actividades escolares de escritura}

La primera pregunta del cuestionario se refiere a las tres actividades de escritura más frecuentes, desde la primaria hasta la educación media superior. ${ }^{8}$ En las respuestas (véase la tabla 2) están los textos escolares destinados a la organización y procesamiento de la información, entre ellos destacan los organizadores gráficos y de recuperación de ideas. Le siguen los académicos, que involucran una reflexión más analítica y argumentada sobre un tema y, finalmente, los textos creativos. Estos últimos tienen una frecuencia elevada (11). Se puede inferir la importancia que a este tipo de textos le confieren los profesores. Lo que queda por indagar es si lo consideran

8 En México, la primaria inicia cuando el niño tiene 6 años de edad y tiene una duración de seis años. Continúa la secundaria, con tres años. Ambos conforman la educación básica. El siguiente nivel es la educación media, que consta de tres años e inicia cuando el joven tiene alrededor de 15 años. Todos estos niveles son obligatorios.

DiáloOos sobre Educación 
una actividad obligatoria como parte de una práctica de géneros textuales o como una forma de expresión que permite el desarrollo de la personalidad y la creatividad.

Tabla 2. Actividades de escritura recurrentes durante el periodo de educación básica y media superior

\begin{tabular}{|c|c|c|c|c|c|c|c|c|c|c|c|}
\hline \multicolumn{8}{|c|}{ Textos escolares } & \multirow{2}{*}{\multicolumn{2}{|c|}{$\begin{array}{l}\text { Escritura } \\
\text { creativa }\end{array}$}} & \multirow{2}{*}{\multicolumn{2}{|c|}{$\begin{array}{c}\text { Textos } \\
\text { académicos }\end{array}$}} \\
\hline \multicolumn{2}{|c|}{$\begin{array}{c}\text { Textos } \\
\text { sintéticos }\end{array}$} & \multicolumn{2}{|l|}{$\begin{array}{l}\text { Opinión } \\
\text { personal }\end{array}$} & \multicolumn{2}{|c|}{$\begin{array}{c}\text { Recuperación } \\
\text { de ideas }\end{array}$} & \multicolumn{2}{|c|}{$\begin{array}{c}\text { Organización de } \\
\text { ideas }\end{array}$} & & & & \\
\hline Resumen & 41 & Reflexión & 2 & $\begin{array}{l}\text { Cuestiona- } \\
\text { rios }\end{array}$ & 9 & $\begin{array}{l}\text { Organizadores } \\
\text { gráficos }\end{array}$ & 9 & Cuento & 11 & Ensayo & 45 \\
\hline Síntesis & 5 & Opiniones & 2 & Paráfrasis & 5 & Reportes & 5 & $\begin{array}{l}\text { Narracio- } \\
\text { nes }\end{array}$ & 8 & $\begin{array}{l}\text { Investiga- } \\
\text { ciones }\end{array}$ & 6 \\
\hline Reseñas & 3 & $\begin{array}{l}\text { Comenta- } \\
\text { rios }\end{array}$ & 2 & Dictados & 4 & Análisis & 5 & $\begin{array}{l}\text { Autobio- } \\
\text { grafía }\end{array}$ & 3 & Critica & 3 \\
\hline Total & 49 & Total & 6 & Total & 18 & Total & 19 & Total & 22 & Total & 54 \\
\hline
\end{tabular}

Fuente: resultados del cuestionario.

De estas actividades, las más significativas para los estudiantes son: el ensayo, el resumen y el cuento, en este orden de frecuencia (véase la gráfica 1). Se les preguntó la razón por la cual este escrito es el más significativo. Sobre el ensayo, los estudiantes respondieron que es una forma de expresión personal y un medio para aprender. No lo conciben como un género que permita la expresión de una idea u opinión desde la disciplina, definición canóniga del género. Esto indica que para ellos el ensayo está enmarcado en el proceso escolar, como una actividad de aprendizaje y no de expresión o reflexión personal. Esta poca independencia de la forma escolar indica una institucionalización de la escritura. Los estudiantes de semestres avanzados consideran al ensayo como parte de sus hábitos académicos pues los ensayos predominan como actividad al final de la carrera.

Gráfica 1

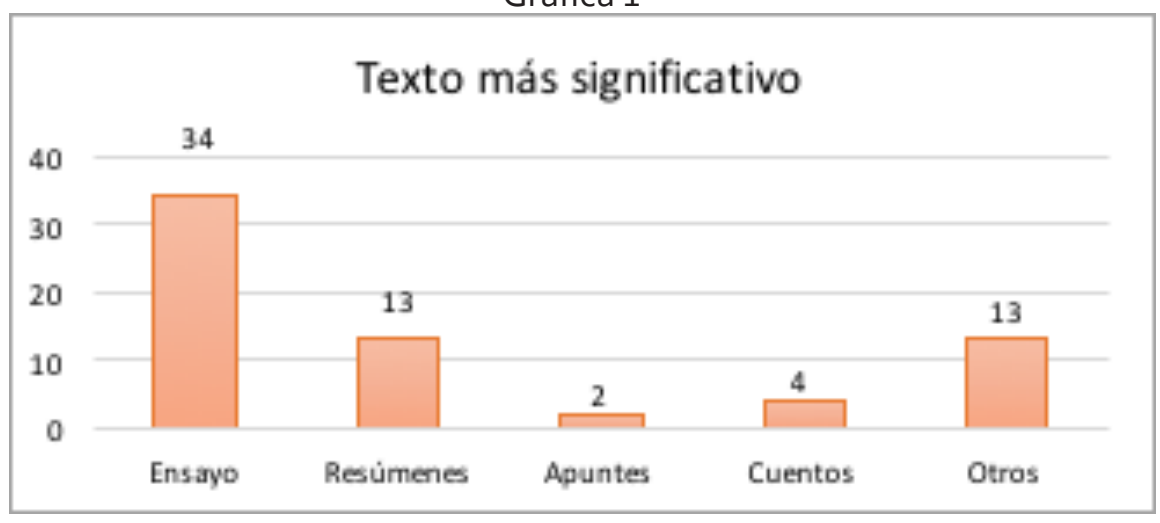

Fuente: Resultados del cuestionario. 
El ensayo es importante para los estudiantes porque les permite:

- Organizar la información para su comprensión. Se diferencia de los organizadores y resúmenes porque en el ensayo se tiene que comprender a profundidad la información, además de argumentar. La expresión del punto de vista personal es fundamental para estos escritores: "te ayuda a organizar las ideas y tus puntos de vista sobre algún tema" (E4). "Porque me permite abordar ideas, argumentarlas, citar el trabajo de otros y finalmente, reflexionar sobre un tema de mi interés" (E12).

- Es un medio para la investigación. Para ellos, este género les exige la investigación como una actividad sistemática para acercarse a un tema de interés. Conceptualizan el ensayo como mediador del aprendizaje: "El ensayo me permitía investigar sobre los temas de mi interés particular y construir un texto propio" (E48), "Porque se tiene que investigar e informarse sobre el tema del ensayo y se aprende a argumentar" (E24), "Es el más significativo porque me ayudó a comprender mejor algunos temas, ya que para realizarlo tenía que investigar mucho" (E26).

- Es un medio de aprendizaje. Este aspecto tiene la mayor recurrencia entre las respuestas (24 de 47). Para ellos, el ensayo es un ejercicio imprescindible para aprender a argumentar y emitir una opinión fundamentada. Asimismo, lo conciben como una manera de adquirir pensamiento crítico. Este punto que reconocen los estudiantes es medular para comprender la razón por la cual son buenos escritores.

La Asociación de Enseñanza Progresista, en un informe conecta "el desarrollo de la habilidad del lenguaje con el aprendizaje en todas las disciplinas [...] y asocia el dominio del lenguaje con el pensamiento crítico. Este nexo entre el lenguaje y el pensamiento crítico pronto se amplió al vínculo entre el lenguaje y los modos disciplinares de pensamiento" (Bazerman et al., 2016: 72-73). La producción de ensayos es la base para la independencia de su pensamiento y su adscripción a la disciplina. Esto también coincide con lo que plantea Ramírez Silva en cuanto a que los estudiantes motivados no están centrados en la calificación, sino en "la comprensión de los temas y al aprendizaje de la redacción" (Ramírez Silva, 2012: 159). En voz de los estudiantes: "Porque para escribirlo tenemos que investigar mucho y tener buenos argumentos que respalden la información" (E47). "En ellos [en los ensayos] se reflejaba qué tanto y qué tan bien habría aprendido. Si no había sido suficiente, en el proceso sabía qué me faltaba" (E13). "Es el que me ha dejado mayores conocimientos, tanto de conocimientos académicos e históricos como de redacción" (E58). En ese tenor, los estudiantes resaltan la planeación, la organización discursiva, la investigación a partir de sus intereses personales y la implementación del pensamiento crítico como los aspectos más significativos del ensayo en el periodo escolar. 
Sigue en importancia el resumen. Es significativo para los estudiantes porque les ayuda a:

- Organizar la información del texto. En este punto encontramos que el resumen les ayuda a: 1. Comprender la información: "Porque desde que estaba en la primaria me dejaban hacerlos, me gustan ya que así aprendo más fácil lo visto en libros o en clase" (E37). 2. Jerarquizar ideas: "Me ayudó a identificar las ideas principales del texto y a organizarlas en un nuevo texto" (E7); "Porque me ayuda a organizar de forma breve lo más importante de algún tema" (E55); y 3. a reinterpretar la información: "Hablaba sobre el tema, pero con mis propias palabras, no tanto con las del autor" (E1).

- Memorizar. Definen la elaboración del resumen como una actividad para recordar lo que se leyó o para retener información por medio de la escritura: "Me es más fácil retener información al escribirla" (E20); y

- El resumen es una herramienta de estudio que facilita el proceso educativo: "Porque es lo que más he hecho y lo que hasta ahora uso para estudiar" (E28);"Porque me ayuda a organizar de forma breve lo más importante de algún tema” (E55);“Porque me ayuda a repasar lo que anteriormente ya había leído" (E41).

Como se observa, el resumen cumple con su objetivo como un texto que sirve de apoyo al proceso de aprendizaje.

Dentro de la escritura creativa, el cuento es el que obtiene más frecuencias (11). Para los alumnos es significativo porque es una forma de expresión personal que propicia la creatividad y la vinculación con la escritura como un objeto de conocimiento de su entorno. Como parte de la expresión personal, de las ideas propias o de la imaginación, puede ser un punto de liberación. Los textos creativos como los cuentos, responden a una práctica conservada de cuando la enseñanza de la escritura estaba ligada a la enseñanza de la literatura, pero también a la producción de diferentes tipos de textos. La identificación de los textos permitirán evaluar las distintas prácticas letradas. Para los alumnos, el cuento ha sido significativo porque: "desarrolla mi capacidad de escribir" (E22); "Por la libertad de expresarme y encontrar formas creativas de expresar; porque me hizo relacionarme y conocer ese género de escritura" (E10); "Me permite expresarme y comunicarme con el mundo" (E56); "Exploraba mi imaginación, escribía con todo tipo de estructuras" (E40) y para expresarse considerando al lector: "porque me obligaba a que me entendieran usando mi imaginación y la buena redacción" (E62). Esta última respuesta ya demuestra una conciencia pragmática del género. A pesar de que el cuento es una actividad escolar, no está ceñida a estructuras académicas como el ensayo o el resumen. Ello les permite expresar sus inquietudes, pensamientos y practicar su creatividad. Si se repasan las respuestas, la creatividad está ligada con el aprendizaje de la escritura, pero también con su libertad creativa y como una forma de interpretar el mundo. 
En este tenor, las actividades de escritura creativa brindan a los estudiantes "la libertad para desarrollar sus pensamientos sin el peso de tener que ajustarse a características superficiales de las convenciones de la escritura académica. Elbow sostiene que "la estructura profunda del discurso académico no difiere de la estructura profunda del buen discurso no académico" (cit. por Bazerman et al., 2016: 186).

El equilibrio entre la escritura de ensayos o resúmenes, así como de textos creativos, permitiría el desarrollo de ideas propias de los sujetos, del dialogismo y del pensamiento crítico, lo que facilitaría que el escritor inscriba sus textos en el ámbito de la disciplina y que, además, sean originales. Por ello estamos de acuerdo con el enfoque llamado Escribir para Aprender como "una alternativa para que lo personal y lo disciplinar interactúen dialécticamente en lugar de que una voz deba ser silenciada para que la otra pueda hablar" (LeCourt, 1996, cit. por Bazerman et al., 2016: 186). Este acercamiento propicia la literacidad desde un enfoque decolonial, porque permite producir discursos propios desde la "historia personal o social y activar la agencia individual y colectiva" (Hernández-Zamora, 2019: 368).

Otra de las preguntas fue sobre la práctica colaborativa de la escritura. A la pregunta de si realizaban textos en talleres antes de entrar a la licenciatura, $36 \%$ dijo que sí y $64 \%$ que no. Para aquellos que participaron en talleres fue una experiencia enriquecedora. Por las respuestas identificamos que, para los profesores, la actividad de escritura en taller está relacionada con la escritura creativa. A los que respondieron que sí habían participado en talleres se les preguntó sobre tres experiencias de aprendizaje. Las respuestas sobre sus aprendizajes fueron diversas. Los conceptos rescatados son: aceptar la crítica, dicción y prosodia, claridad, tipología textual, corrección, argumentación, elaboración de textos, interacción con compañeros, respeto, confianza, mejorar la lectura, aportación de nuevas lecturas, investigación, aprender a retroalimentar y a estructurar un texto e incrementar la creatividad.

Ante estas respuestas se observan los distintos niveles y tipos de aprendizajes. Lo que resalta es la retroalimentación (que se analizará en el punto siguiente) y aspectos actitudinales como aceptar la crítica, el respeto y la confianza: "Respetar lo que el otro hace es esencial" (E8); "Aprendí a aceptar positivamente las críticas constructivas" (E49); "La lectura en voz alta de los textos que te va quitando el miedo al 'qué dirán"' (E64). De igual forma, se percibe la importancia de la interacción con los compañeros y la expresión de las ideas propias: "[aprendí] a hacer textos exponiendo mi opinión sobre algo" (E60); "Escribir textos en grupo; compartir lo que escribo" (E59).

Las actividades colectivas propician la formación de comunidades de práctica o, como se han definido en el Modelo educativo mexicano: prácticas sociales del lenguaje (Secretaría de Educación Pública, 2011, 2020). El concepto de práctica es amplio, tanto para la realización de tareas como para aprendizajes de diferente orden, por lo que: 
dentro de una comunidad de práctica, todos sus miembros acumulan una diversidad de valores, normas, modelos culturales y narrativas tácitos y dados por sentado como parte de su socialización y de su inmersión continua en la práctica. Aceptar tácitamente estos valores, normas, modelos culturales y narrativas (en la mente, en la acción y en la práctica incorporada) y compartirlos con los demás es precisamente lo que significa ser miembro de la comunidad de práctica (Gee, 1999: 10, la traducción es nuestra).

Entonces, los resultados obtenidos en esta parte de nuestra investigación permiten replantear la necesidad de trascender la escritura como una práctica escolar, centrada en procesos individuales de alfabetización, hacia una perspectiva social y como parte de una sociedad letrada. Asimismo, subrayamos que la escritura colaborativa puede ser también académica, no solo creativa.

\section{Revisión de la escritura}

La retroalimentación en los trabajos es sustancial para el proceso de escritura. No se puede aprender a escribir si no hay una guía que marque el nivel de legibilidad de los textos. Es un paso fundamental dentro del proceso. Una vez que se domine la competencia, el escritor puede distanciarse del texto e implementar la autorrevisión del borrador. Los profesores deberían practicar la corrección de los textos de los alumnos y la corrección entre pares para retroalimentar sus avances y propiciar la formación autocrítica.

A la pregunta sobre si los profesores les revisaban sus textos escritos, 66 de 67 respondieron que sí. Esto indica una favorable expectativa hacia el proceso educativo de los estudiantes. Indica que hubo un proceso de diálogo pedagógico. Sin embargo, las respuestas sobre el tipo de retroalimentación recibida matizan estos resultados. Las respuestas de los estudiantes sobre el tipo de retroalimentación que recibían pueden agruparse en: observaciones sobre ortografía, sintaxis, discurso y aspectos motivacionales. Ocho no respondieron y doce dieron respuestas imprecisas que no tienen que ver con la escritura o solo como una anotación de "revisado".

Las respuestas que anotaron aspectos de sintaxis, gramática o puntuación, se categorizaron como cohesión. Cassany (2000) la define como la organización del enunciado entre sus propios elementos (cohesión léxica) y su vinculación o encadenamiento con otros enunciados (cohesión textual). Los alumnos de semestres avanzados ya identificaron las observaciones de sus profesores como cohesión.

Las respuestas sobre la organización y estructura global del texto, así como el sentido del mensaje, se categorizaron dentro de la coherencia. Definida por Cassany (2000) como la selección de información, su organización y la estructura del texto, es la organización discursiva global del texto más difícil de lograr pues implica una relación lógica argumentada entre las temáticas, lo que se desea expresar y el género textual. En coincidencia con los textos recurrentes solicitados por los profesores, tales como el ensayo, el cuento y el resumen, es de esperarse que la revisión de la cohesión y la coherencia predominen como parte de la retroalimentación. 
La motivación como parte de la retroalimentación es importante para los estudiantes pues los alienta a mejorar o resalta su potencial como escritores. Los estudiantes buscan el reforzamiento de su conducta, una de las funciones de la retroalimentación que apunta Kulhavy (1977, en Tapia-Ladino, 2014).

Por los resultados, la revisión es una práctica escolar frecuente, sin embargo, predomina la importancia de la revisión ortográfica (20 frecuencias) sobre aspectos discursivos o emotivos.

Para los estudiantes, el reconocimiento de sus capacidades de escritura es un aspecto significativo en la revisión. A la pregunta sobre lo que les gustaría que les revisaran, lo que menos les interesa que les revisen es la ortografía, contrariamente a lo que hacen los profesores, según sus propias respuestas. Ellos quieren que se les revise la cohesión, la coherencia, la argumentación y la pragmática en sus textos. Estas son algunas de sus respuestas: "Lo principal que quisiera que revisaran es la concordancia, la sintaxis. Me gustaría saber si estoy redactando bien. La segunda cuestión de la que quisiera retroalimentación es sobre la manera de desarrollar los textos, muchas veces no creo llevar un orden coherente de ideas" (E63); "Sobre la estructura; de acuerdo a si redacto correctamente y qué mejorar" (E18); "La estructura de los textos. Recursos utilizados que son innecesarios" (E23).

Les interesa mejorar su producción de ideas, ya que apuntan que les gustaría que les recomendaran otros textos. A algunos otros también les gustarían comentarios para mejorar el estilo. Uno apunta que no estaría mal una "felicitación de vez en cuando" (E14). Las respuestas que centran la revisión en aspectos ortográficos, de puntuación y de sintaxis, son principalmente de estudiantes de primer ingreso. No está exenta en ellos la preocupación por la estética del texto. Las respuestas más elaboradas son de los estudiantes de octavo y noveno semestre. Aquí algunos ejemplos sobre lo que les gustaría ser retroalimentados: "[comentarios] sobre el desarrollo argumentativo; sobre lo novedoso o no del tratamiento del tema; aspectos de comprensión lectora: Fui claro o no." (E6); "Comentarios de opinión (sí, no y por qué). Recomendaciones de lecturas" (E23); "Que mi concordancia es correcta. Que mi sentido o mi estilo es muy bello. Que se entiende bien la idea" (E25); "Que mis escritos tuvieron impacto en el lector. Que tienen influencias de mis escritores favoritos. Que la musicalidad es buena" (E49).

Los resultados coinciden con los que obtiene Rogers (2008, en Tapia-Ladino, 2014) en su estudio con 150 estudiantes de la Universidad de Stanford sobre el aprendizaje de la escritura académica. Ellos señalan que "el feedback los ayuda a sincronizar su escritura para ajustarse a la situación retórica y a las expectativas del lector. Tal esfuerzo les permitió dar saltos críticos en el desarrollo de sus habilidades de escritura" (Tapia-Ladino, 2014: 261). De esta manera, sus respuestas sobre lo que esperan de la retroalimentación resaltan ya su formación o inclinación hacia el campo de la lengua y la literatura: aspectos técnicos-discursivos, de estilo y estéticos. 


\section{Oralización de la escritura}

Pocos trabajos han estudiado la oralización de la escritura dentro del proceso educativo. En esta investigación se entiende la oralización como un momento del proceso de la escritura que consiste en reflexionar, organizar ideas, distanciarse del texto escrito para mejorarlo. Esta interacción entre el habla y la escritura influye en el aprendizaje.

Hay que diferenciar la lectura en voz alta de la oralización de la escritura. Si bien es cierto que la lectura en voz alta es una práctica escolar frecuente, también es cierto que ya desde finales del siglo XIX y principios del XX se consideraba importante para la comprensión de textos o para la lectura explicada (Viñao, 2002). La oralización del texto escrito es una actividad más que permite alcanzar otros niveles de comprensión del propio proceso de escritura. Debe diferenciarse la lectura en voz alta, actividad que puede realizarse solo para verificar el cumplimento de la tarea, de la oralización del texto escrito como una actividad reflexiva. La primera ayuda a aspectos orales y de seguridad personal; la segunda, propicia un distanciamiento del texto que permite escuchar la propia voz interna.

La lectura en voz alta es una práctica predominante en de la educación básica y media, como se observa en la gráfica 2.

\section{Gráfica 2}

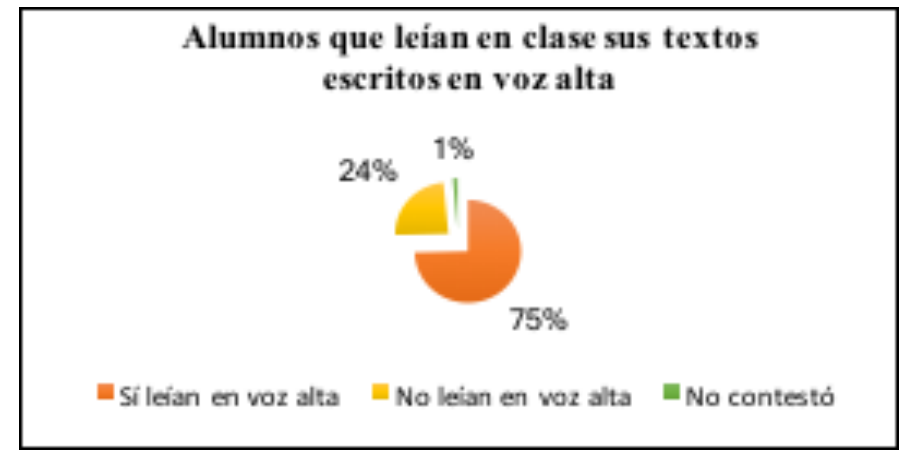

Fuente: resultados del cuestionario.

Sin la discusión sobre lo leído, esta actividad queda a nivel de exposición, no banal, pues compartir los textos en público contribuye al desarrollo de otras habilidades que tienen que ver con el lenguaje oral, la retórica, la organización textual, la retroalimentación grupal y la seguridad personal. La lectura en voz alta de lo escrito es un acto narrativo que hace inteligible un texto, fortalece el aprendizaje, fortalece la seguridad de la persona y centra la posible multiplicidad de significados para lograr la tematización en la escritura (Mier Garza, 2009).

Dentro del proceso de escritura, poco más de la mitad de los encuestados (52\%) afirmaron que leían en voz alta sus textos escritos (véase la gráfica 3). En la gráfica 2 el porcentaje de 
lectura en voz alta es más alto, pues se realiza bajo la dirección de un profesor (75\%). La lectura en voz alta como una oralización es un proceso personal aunque sea público, es decir, incita a la reflexión individual. La lectura y la escritura son parte de un mismo proceso, por el cual el "trabajo de revisión y corrección textual que se hace con la escritura tiene un correlato con la reformulación y la corrección que el hablante aplica durante su actuación oral" (Mostacero, 2011: 115). En este proceso hay "una reformulación discursiva que el hablante ejecuta a partir de su escucha y de la conciencia sintáctica que como enunciador hace dentro de la construcción de su discurso" (Mostacero, 2011: 116).

Gráfica 3

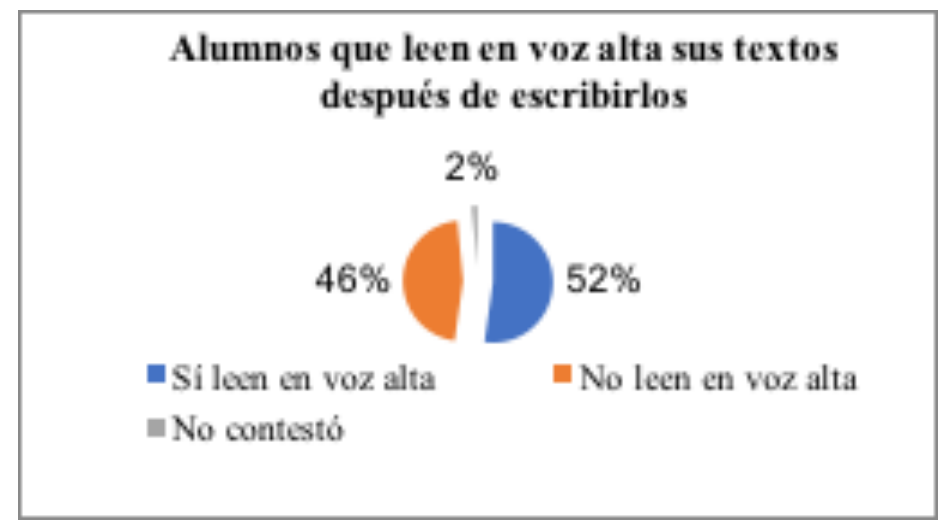

Fuente: resultados del cuestionario.

Esta formulación permite repensar el texto escrito, precisamente porque exalta la conciencia sintáctica, la organización discursiva y el contenido del texto. Investigaciones sobre la escritura a través del currículo muestran distintos aprendizajes logrados mediante las interacciones entre lo oral y lo escrito. Basadas estas en el concepto de dialogismo derivado de las teorías de Bajtin, y de Vygotsky principalmente, la interacción oral entre profesor y alumno favorece la participación, y con ello, la mayor diversidad de voces (Dysthe, 1996 en Bazerman et al., 2016). Las participaciones orales y escritas son consideradas como herramientas para pensar. Otro estudio encontró que los resultados de estas interacciones cumplen funciones distintas para los estudiantes: los "más versados pueden reunir información por su cuenta para profundizar y consolidar su conocimiento, mientras que los estudiantes menos versados requieren apoyo para reunir y comprender la información (Rivard, Straw, 2000 en Bazerman et al., 2016: 104).

Keys (1999, en Bazerman et al., 2016) utilizó con estudiantes de ciencias en secundaria la lectura en voz alta ${ }^{9}$ para propiciar el razonamiento en el aprendizaje de contenidos disciplinares. Los aprendizajes fueron diferenciados, centrados en la planificación retórica, en la secuencia textual y en la resolución de problemas.

9 Lo que entiende este autor por lectura en voz alta es lo que nosotros definimos como oralización de la escritura. 
La oralización de los argumentos contenidos en los textos escritos no es frecuente. Según las respuestas de los encuestados, solo $37 \%$ lo hacían (véase la gráfica 4). Sin embargo, ya aparece. Esto puede incorporarse también en la medida que los profesores se sientan capacitados para manejar este tipo de prácticas.

\section{Gráfica 4}

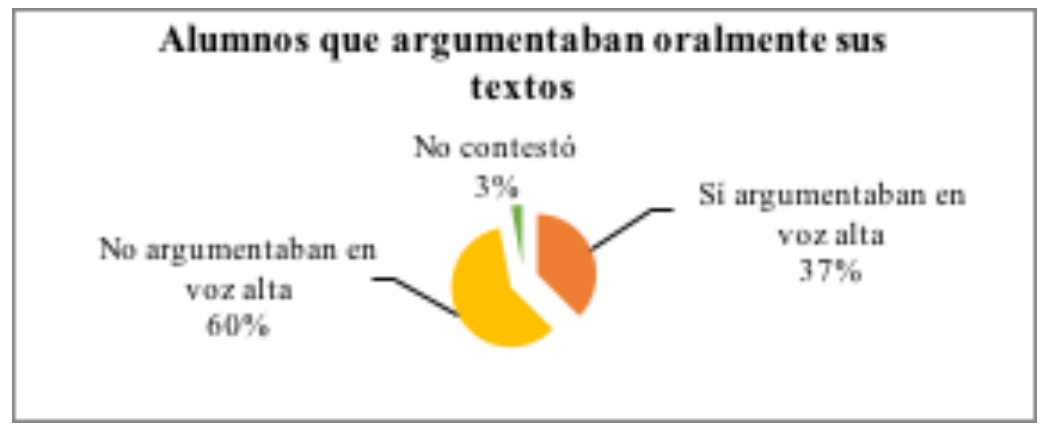

Fuente: resultados del cuestionario

Sobre los tres aprendizajes obtenidos con la oralización de sus textos las respuestas fueron variadas, tanto que fue difícil realizar la reducción conceptual. Entre las menos mencionadas está el aprendizaje sobre la coherencia, el estilo y la retroalimentación. Luego están las respuestas que podríamos ubicar en el nivel discursivo: aprendizajes sobre la redacción, cohesión y sentido. Este último, también relacionado con la comprensión del texto por los receptores. El distanciamiento del texto lo reconocen como un aprendizaje: "Corrección desde mi propia voz" (E4); “Obtener otra perspectiva de mi texto” (E18); “La reflexión de lo escrito” (E15).

Los aprendizajes más mencionados son la prosodia, la dicción y la puntuación. La prosodia entendida como la acentuación, entonación, ritmo y cacofonía (aspectos suprasegmentales del discurso) (Quilis, 1993). La lectura en voz alta les sirve para encontrar "La entonación adecuada para enfatizar; buscar y generar un ritmo para el texto" (E16). La dicción, definida como la modulación de la voz según el Diccionario de la Real Academia. Para ellos, la pronunciación les permite: "Leer bien en voz alta (claro)" (E30); "Vocalizar adecuadamente" (E39); "Modular la voz" (E52). El caso de la puntuación tiene que ver tanto con aspectos de prosodia como con la puntuación gráfica del texto. Este aspecto todavía permeado por la premisa de que las comas y puntos indican pausas en la lectura.

Un punto que resaltar es que 13 de los encuestados respondieron que este ejercicio les dio seguridad: "Se vence a la vergüenza" (E22); "el pánico escénico se quita poco a poco" (E64); "Confianza" (E33 y E34). Otras respuestas muestran distintos grados de reflexión sobre el texto escrito: "es una experiencia casi liberadora" (E46); "permite apropiarme del texto" (E47); "Obtener otra perspectiva de mi texto" (E18). Estas últimas respuestas muestran el grado de reflexión y 
relación epistémica con el texto escrito, que va más allá de una mera forma de comunicación escolar. En suma, predomina todavía la lectura en voz alta como apoyo para mejorar aspectos suprasegmentales. La oralización de la escritura, la cual incide en mejorar aspectos discursivos, reflexivos o argumentativos, todavía es una práctica pendiente de incorporarse para alcanzar el distanciamiento epistémico sobre el discurso y la postura argumentada frente un tema.

\section{Discusión de los resultados}

Las prácticas de escritura en educación básica y media superior que contribuyeron a formar a los estudiantes como escritores competentes son la producción de textos escolares, académicos y creativos. El trabajo colaborativo en taller y la revisión pueden considerarse prácticas dialógicas que apoyaron fuertemente el proceso. La oralización del texto escrito es una práctica poco común, pero con enormes potencialidades para el proceso educativo. Estas prácticas pudieron cuantificarse y clasificarse en los resultados, pero los aspectos más interesantes los encontramos en las respuestas a las preguntas abiertas. Estas ayudan a comprender los significados que los estudiantes dan a los procesos de aprendizaje sobre las prácticas de escritura. En suma, encontramos que los textos que producen los estudiantes en la escuela son los que tradicionalmente se solicitan, estas son prácticas dominantes que adquieren cierta representación en el imaginario de los estudiantes como actividades que corresponden a la escritura escolar.

Para Barton y colaboradores (2000), las prácticas letradas vernáculas tales como escribir diarios, recados o poemas que se realizan en espacios privados o para fines personales, no tienen tanto valor para los estudiantes porque no están institucionalizadas. Sin embargo, para los estudiantes de Letras hispánicas sí son significativas. De los 67 encuestados, 50 escriben textos creativos, ensayos, reflexiones, diarios, reseñas motu proprio. Estos estudiantes, como escritores competentes, combinan las prácticas letradas vernáculas con las prácticas letradas dominantes, lo cual genera una dinámica entre la escritura como empoderamiento, como conocimiento del mundo y su actuación sobre él. Los estudiantes manifestaron esa satisfacción y libertad al escribir. Quienes no logran este equilibrio corren el riesgo de reproducir las prácticas dominantes, lo que pone de manifiesto el alcance de las estructuras pedagógicas de poder (Vargas Franco, 2015).

Los estudiantes de Letras hispánicas son sujetos letrados que están conscientes de la importancia de la revisión y del trabajo colaborativo para fomentar el diálogo con el texto. Este tipo de prácticas rompe con la educación unidireccional y abre la posibilidad de que la enseñanza de la escritura se convierta en un proceso democrático y dialógico. Los estudiantes encuestados manifestaron que esperan que sus textos tengan un efecto sobre el lector (acto ilocutivo), así como también esperan que el profesor emita una opinión sobre el texto entregado (acto perlocutivo) y que esta opinión no se centre en lo académico sino que también sea motivante.

La conciencia del escritor sobre sus posibles lectores, los efectos que desea lograr con su texto, muestra actitudes interactivas que facilitan los procesos de escritura porque se tiene un 
control sobre ella. Las prácticas escolares de escritura previas a las que estuvieron expuestos los estudiantes de esta investigación, los define como sujetos letrados, que podrán realizar una reflexión sobre su entorno y actuar sobre él.

A partir de los resultados, nos percatamos que queda pendiente un análisis que permita describir el tipo de retroalimentación enfocada en la reafirmación de la conducta como una forma de motivación, además del tipo de diálogo que se presenta: correcciones de forma, de fondo, recomendaciones, discusiones sobre lo escrito. Incluir estos aspectos permitirá comprender mejor las distintas literacidades presentes en las prácticas escolares de escritura.

Es de notar el peso que le dan los estudiantes a la escritura como un proceso colaborativo. Los procesos de aprendizaje colectivos permiten el diálogo y la creatividad; la creatividad empodera, elimina el egocentrismo porque se puede visualizar y percibir la comprensión desde el punto de vista de los lectores, además de contribuir en la negociación entre los escritores y sus contextos (cfr. Vargas Franco, 2015). Los resultados obtenidos en este estudio corroboran estos principios que establecen los estudios de literacidad. Los estudiantes manifiestan vetas creativas importantes y la necesidad de ser escuchados y leídos. Asimismo, su desempeño sobresaliente como estudiantes fortalece la afirmación sobre que "una práctica pedagógica que proporcione el intercambio con textos significativos y variados permitirá el desarrollo efectivo de la competencia tanto lingüística como cognoscitiva." (Fraca de Barrera, 1997: 112). Igualmente, diversas prácticas de escritura fomentan la democracia y la liberación de la persona, tal y como lo asientan los NEL en sus principios.

Un proceso recomendado en la enseñanza de la escritura es su oralización. Después de una revisión sobre el tema en distintas bases de datos y revistas especializadas, nos percatamos que este no es un tema muy estudiado desde enfoques de los NEL, pero sí es abordado por investigaciones desde el movimiento de la WAC. En los trabajos encontrados sobre la oralización de la escritura se pueden identificar dos momentos: la lectura pública, a través de talleres, y la lectura privada del texto escrito. La primera propicia el aprendizaje colaborativo, el diálogo y la argumentación, pues el discurso oral exige una planificación al mismo tiempo que la realización lingüística (performancia), además de la seguridad del escritor en sí mismo (Mier Garza, 2009). La segunda permite el distanciamiento del escrito para llegar a una reflexión objetiva. Este continuum entre la oralización y la escritura es una práctica escolar que necesita resignificarse a favor del aprendizaje colaborativo, de la reflexión crítica y de la seguridad del sujeto.

En general, los resultados de este estudio resaltan que la escuela está incorporando prácticas de escritura más complejas, como el ensayo, que combina con textos escolares como apoyo a la adquisición del conocimiento. La copia, como una actividad escrita, ya no aparece entre las prácticas dominantes reportadas por los estudiantes. Su percepción sobre los procesos de revisión y sus reflexiones sobre su aprendizaje demuestran que estos estudiantes significan la escritura como una práctica discursiva. Su escritura puede considerarse un producto social con- 
textualizado. Sus reflexiones sobre la escritura son profundas, diversas y complejas, por lo que es necesario un estudio cualitativo que ayude a definir las conceptualizaciones de los estudiantes sobre la escritura y poder caracterizar su episteme.

La variedad de respuestas de los estudiantes sobre los aprendizajes, y sus expectativas acerca de la retroalimentación, indican que los aprendizajes son diversos a partir de las características, intereses y sus propias reflexiones, por lo que la educación debería considerar la diversidad de formas de aprendizaje e intereses de los estudiantes para diseñar didácticas que no igualen prácticas didácticas, sino que atiendan los intereses de los sujetos y desarrollen las competencias y habilidades particulares. Por otro lado, de las variadas respuestas sobre las actividades en taller o sobre la revisión, podemos inferir que existe poca sistematización, planeación u objetivos claros en las actividades de los profesores. Esta puede ser una oportunidad para abrir los procesos educativos a distintas posibilidades de aprendizaje desde la literacidad.

Finalmente, los resultados describen la complejidad de la escritura desde una dimensión individual y social, que en conjunto explican la construcción de comunidades de escritura, así como las prácticas letradas realizadas a través del paso de los estudiantes por el sistema educativo.

\section{Referencias}

Aguilar, L. E.; G. Fregoso (2013). La literacidad académica en estudiantes de posgrado. Límites y principios de una práctica académica. Ponencia, XII Congreso Latinoamericano para el Desarrollo de la Lectura y Escritura. México: Consejo Puebla de Lectura, A.C.

Barton, D.; M. Hamilton; R. Ivanic (2000). Situated Literacies. London: Routledge.

Bazerman, C.; J. Little; L. Bethel; T. Chavkin; D. Fouquette; J. Garufis (2016). Escribir a través del curriculum. Una guía de referencia. Argentina: Parlor Press LLC.

Carlino, P. (2004). El proceso de escritura académica: cuatro dificultades de la enseñanza universitaria. Educere, 8(26), 321-327.

Casillas Padilla, E. (2018). La redacción académica en la licenciatura en Letras hispánicas de la Universidad de Guadalajara. Narrativas oficiales, estudiantes y docentes. Tesis de maestría. México: Instituto Superior de Investigación y Docencia para el Magisterio.

Cassany, D. (2000). La cocina de la escritura. Papeles de Pedagogía, (42). Barcelona: Paidós.

Cervantes Velázquez, M. R. (2015). Procesos de escritura académica. Un acercamiento con estudiantes universitarios. México: Universidad Autónoma de Aguascalientes.

Flórez Romero, R.; M. C. Torrado Pacheco; I. Arevalo Rodríguez; C. Mesa Guecha; S. Mondragón Bohorquez; C. Pérez Venegas (2005). Habilidades metalingüísticas, operaciones metacognitivas y su 
relación con los niveles de competencia en lectura y escritura: un estudio exploratorio. Forma y Función, 15-44. https://revistas.unal.edu.co/index.php/formayfuncion/article/view/17975

Fraca de Barrera, L. (1997). La lectura y la escritura como procesos psicosociolingüísticos: una aproximación pedagógica. En Martínez, M. C. Los procesos de la lectura y la escritura. Colombia: Universidad del Valle, 107-128.

Gee, J. P. (1999). The New Literacy Studies: From 'Socially Situated' to the Work of the Social. En Barton, D.; M. Hamilton; R. Ivanic (eds). Situated Literacies. Reading and Writing in Context. Londres: Routledge, 180-196.

Hernández Sampieri, R.; R. Fernández-Collado; P. Baptista Lucio (2006). Metodología de la investigación, México: Mc Graw Hill.

Hernández-Zamora, G. (2019). De los Nuevos Estudios de Literacidad a las perspectivas decoloniales en la investigación sobre literacidad. Ikala. Revista de lenguaje y cultura, 24(2), 205456. https://doi.org/10.17533/udea.ikala.v24n02a10

Margarito Gaspar, M.; S. Quezada Camberos; L. E. Aguilar González. (2013). Las competencias de escritura en los alumnos de primer ingreso de la licenciatura en Letras Hispánicas de la Universidad de Guadalajara. Ponencia, XII Congreso Latinoamericano para el Desarrollo de la Lectura y Escritura. Puebla, México: Consejo Puebla de Lectura, A. C.

Martínez, M. C. (1997). Los procesos de la lectura y la escritura. Colombia: Universidad del Valle.

Mier Garza, R. (2009). La multiplicidad de voces: la narración como juego de vínculos. En Contreras Islas, I.; A. D. García Collino. Escritos sobre oralidad. México: Universidad Iberoamericana, 13-40.

Molina-Natera, V. (2012). Escritura a través del currículo en Colombia: situación actual y desfíos. Magis. Revista Internacional de Educación, 5(10), 93-108. https://www.redalyc.org/articulo.oa?id=2810/281024896006

Mostacero, R. (2011). Oralidad, escritura y escrituralidad. Enunciación, 16(2) 100-119. https://revistas.udistrital.edu.co/index.php/enunc/article/view/3908/14508

Quilis, A. (1993). Tratado de fonología y fonética españolas. Madrid: Gredos.

Ramírez Silva, A. (2012). Los estudiantes y la escritura universitaria. México: Universidad Pedagógica Nacional. Horizontes educativos.

Secretaría de Educación Pública (2011). Acuerdo número 592 por el que se establece la articulación de la educación básica. México: Secretaría de Educación Pública.

http://edu.jalisco.gob.mx/cepse/sites/edu.jalisco.gob.mx.cepse/files/acuerdo 592web.pdf

Secretaría de Educación Pública (2011). Plan y programas de estudio 2011. Primaria. México: Secretaría de Educación Pública. https://www.planyprogramasdestudio.sep.gob.mx/introcampo-lengua.html

Soriano, M. (2001). La motivación, pilar básico de todo tipo de esfuerzo. Revista de Relaciones Laborales, (9), 163-184. 
Tapia-Ladino, M. (diciembre de 2014). Los comentarios escritos: género orientado a la consecución de otro género en el proceso de escritura académica. Onomázein, (30), 254-268. http:// onomazein.letras.uc.cl/Articulos/N30/30 21 TapiaFINAL.pdf

Vargas Franco, A. (2015). Escritura académica e identidad en la educación superior: un enfoque sociocultural. Colombia: Fondo editorial ITM.

Viñao, A. (2002). La enseñanza de la lectura y la escritura: análisis sociohistórico. Anales de documentación, 345-359. I Congreso Nacional de Lengua Escrita (1999).

https://docplayer.es/9409208-La-ensenanza-de-la-lectura-y-la-escritura-analisis-socio-historico.html

Zavala, V. (2011). La escritura académica y la agencia de los sujetos. Cuadernos Comillas, (1), 5266. https://docer.com.ar/doc/snx08c

\section{Anexo \\ Cuestionario sobre prácticas escolares de escritura}

\section{Actividades escolares de escritura}

1. Según tu experiencia, escribe las tres actividades de escritura más recurrentes (desde primaria hasta preparatoria) que realizaste en el aula.

2. De las tres que escribiste, escoge la más significativa para tu proceso de aprendizaje.

3. ¿Por qué es la más significativa?

4. En grados anteriores a la licenciatura (de primaria a preparatoria) ¿alguna vez trabajaste textos escritos en taller?

o Sí o No

5. Si trabajaste en taller, escribe tres experiencias de aprendizaje.

6. Además de las actividades escolares ¿escribes o escribías por tu cuenta?

o Sí o No

\section{Revisión de la escritura}

7. ¿Tus profesores revisaban tus textos escritos? (Se considera revisión que realizaran alguna anotación, no solo la calificación).

o Sí O No

8. ¿Qué tipo de observaciones realizaban?

9. ¿Qué tipo de observaciones te gustaría que realizaran? Escribe tres.

\section{Oralización de la escritura}

10. Una vez que terminas de escribir: ¿lees en voz alta tus textos?

o Sí o No 
11. En la escuela ¿alguna vez los profesores solicitaron que leyeras en voz alta tus escritos? o Sí O No

12. Escribe tres aprendizajes que hayas tenido con la lectura en voz alta de tus textos.

13. En grados escolares anteriores a la licenciatura ¿alguna vez durante las clases discutiste oralmente con argumentos lo que escribías?

o Sí O No

14. Si alguna vez trabajaste en taller ¿se les solicitaba que expusieran oralmente sus ideas? O Sí O No

15. Si respondiste que sí ¿cuál es tu opinión acerca de esta actividad? 